\title{
Interação entre sensores piezoelétricos e microcontroladores para confecção de um tambor eletrônico MIDI
}

\author{
Oliveira, V. S. P. P. ${ }^{1^{*}}$; Monjardim E. J. Z. ${ }^{2}$; Galgano, G. D. ${ }^{3}$ \\ ${ }^{1}$ Graduação em Licenciatura em Física, Universidade Federal do Espírito Santo, Alegre, ES, Brasil. \\ ${ }^{2}$ Graduação em Ciência da Computação, Universidade Federal do Espírito Santo, Alegre, ES, Brasil. \\ ${ }^{3}$ Departamento de Química e Física, Universidade Federal do Espírito Santo, Alegre, ES, Brasil.
}

* e-mail: vitor.s.oliveira@edu.ufes.br

\begin{abstract}
Resumo
Microcontroladores são dispositivos equipados com uma unidade de processamento, que em combinação com sensores, são capazes de realizar experimentos físicos. Nesse trabalho nós construímos um tambor de bateria eletrônica de duas zonas, utilizando sensores piezoelétricos como triggers controlados por uma placa Arduino. Fizemos experimentos para caracterizar os sensores e otimizar a sua integração a um tambor equipado com uma pele muda, de forma que o tambor não emite som expressivo quando atingido pela baqueta, mas torna-se um controlador MIDI percussivo. O resultado é um sistema econômico, estável e funcional para a prática e execução musical, sendo também de interesse para o estudo da Física Aplicada.
\end{abstract}

Palavras chaves: sensores, microcontroladores, efeito piezoelétrico, MIDI, percussão.

\begin{abstract}
Microcontrollers are devices equipped with an integrated circuit, which, in tune with sensors, are capable of carrying out physical experiments. In this work we built an electronic drum with two zones, using piezoelectric sensors as triggers controlled by an Arduino board. We did experiments to characterize the sensors and optimize their integration to a drum equipped with a muted skin, so that the drum doesn't emit an expressive sound when hit by a stick, but becomes a percussive MIDI controller. The result is an economical, stable and functional system for musical practice and performance, which is also of interest for the study of Applied Physics.
\end{abstract}

Keywords: sensors, microcontrollers, piezoelectric effect, MIDI, percussion.

\section{Introdução}

Neste artigo, foi explorada a integração de sensores analógicos com microcontroladores, investigando a implementação desse tipo de sistema na área da produção musical. Elaboramos um tambor de bateria eletrônica de duas zonas, identificadas pela membrana e 0 aro, utilizando sensores piezoelétricos como triggers controlados por uma placa Arduino. $\mathrm{O}$ tambor eletrônico simula um tambor acústico, porém a diferença é que ele não emite sons próprios, simulando sons de um tambor real com amostras de áudios, executadas no computador através de um software.

O sensor utilizado foi do tipo piezoelétrico, é caracterizado por um elemento de transdução feito de material piezoelétrico. $\mathrm{O}$ efeito piezoelétrico ocorre quando uma deformação mecânica dá origem a uma polarização de cargas, de forma que uma corrente elétrica no interior do material [1]. Uma vez tendo os sensores piezoelétricos acoplados a estrutura do instrumento, os mesmos são ligados a um microcontrolador Arduino que por sua vez é ligado ao computador, pela porta USB. Quando uma das sessões do tambor sofre impacto, as vibrações resultantes são captadas pelos sensores que emitem um sinal elétrico proporcional captado na porta analógica do Arduino. No Arduino o código de processamento interpreta esse sinal análogo e converte para sinal digital que é enviado para saída USB do Arduino, é interpretado pelos softwares instalados no computador e uma série de programas associa esse sinal a uma nota musical e em resposta reproduz um som associado a uma das partes do tambor.

O resultado é um sistema estável e funcional para a prática e execução musical, sendo de interesse para o estudo do hardware e também da física aplicada, envolvida no efeito piezoelétrico. Sua principal vantagem é sua prática silenciosa, produzindo menos ruído do que tambor tradicional, sendo ideal para estudos. Usamos a plataforma Arduino, pois a própria 
facilita a concepção e a realização de experimentos de Física por disponibilizar o software e o hardware que permitem o controle e o processamento de dados obtidos a partir de sensores externos, ou mesmo em suas portas I/O [2].

\section{Materiais e métodos}

\subsection{Sensores piezoelétricos}

O sensor é composto basicamente por um disco de latão, pastilha de cerâmica e eletrodo de prata [3]. A aplicação uma pressão sobre o sensor, ocasiona uma diferença de potencial proporcional. O sinal do sensor piezoelétrico passa por um circuito eletrônico de condicionamento, que irá filtrar e amplificar o sinal de forma que este tenha uma relação sinal/ruído alta, facilitando a leitura, além de tornar a resposta do sensor linear em uma faixa de trabalho ampla [4].

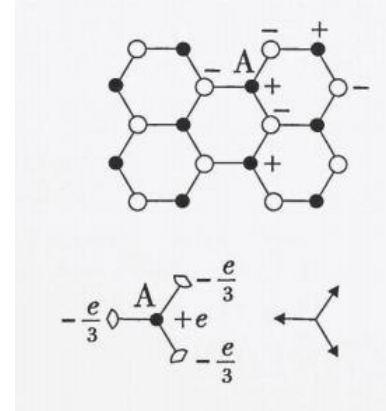

(a)

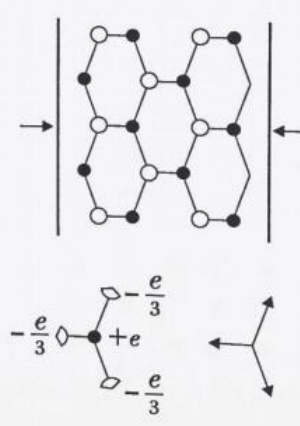

(b)
Figura 1: (a) Cristal sem deformação onde o momento de dipolo elétrico total é nulo. (b) Cristal com deformação onde a resultante dos momentos de dipolos elétricos não é nula.[1]

Com relação ao ponto de vista da física do material, os materiais piezoelétricos apresentam uma polarização local de carga, ou seja, uma separação entre cargas positivas e negativas. Contudo essas cargas positivas e negativas são simetricamente distribuídas no volume do material, de onde de um ponto de vista global, o material fica eletricamente neutro. Quando uma tensão mecânica é aplicada em uma direção, tal que, a simetria da distribuição de cargas é perturbada, o campo elétrico não é mais nulo, e surge uma diferença de potencial proporcional a tensão aplicada [1]. A situação pode ser ilustrada a figura 1, adaptada de [1], na Figura 1a temos um cristal hexagonal ideal inicialmente sem deformação, onde a soma de todos os dipolos elétricos é nula, em razão de simetria, mas quando essa simetria é perturbada por uma tensão mecânica externa o momento de dipolo elétrico resultante deixar ser nulo, e dessa forma o material fica polarizado resultando em um campo elétrico.

\subsection{Hardware}

Os sensores foram instalados em duas zonas do Tambor (Figura 3, à esquerda). Uma responsável por captar as vibrações da membrana e a outra pelo aro. Cada vez que uma das partes do instrumento são tocadas, as vibrações causadas pelo impacto são captadas pelos sensores. Eles emitem um sinal elétrico ao qual é recebido pelas portas analógicas do microcontrolador, esses sinais são captados e interpretados pelo algoritmo programado no Arduino. Esse sinal analógico é convertido para digital e interpretado pelos softwares instalados no computador, ao qual o microcontrolador é conectado, e em resposta reproduz um som associado a uma das partes do instrumento.

\subsection{Software}

Para confecção do sinal, foram utilizados softwares livres. O primeiro deles é o próprio software proprietário da placa Arduino, utilizado para programar o microcontrolador, ArduinolDE. Inicialmente, os sinais dos sensores são interpretados, pré-processados, digitalizados e convertidos para formato MIDI. O sinal de saída é enviado para a porta USB, que conecta o microcontrolador ao computador. O Hairless MIDI faz a leitura da porta USB e envia o sinal para a porta MIDI. Essa porta MIDI é criada pelo loop-MIDI que ao mesmo tempo faz a leitura da porta MIDI e a envia para um software de sonorização, como, por exemplo, o Hydrogen, que faz a leitura do MIDI e reproduz o som previamente escolhido para determinado tipo de sinal, dentre os sons disponíveis no banco de dados.

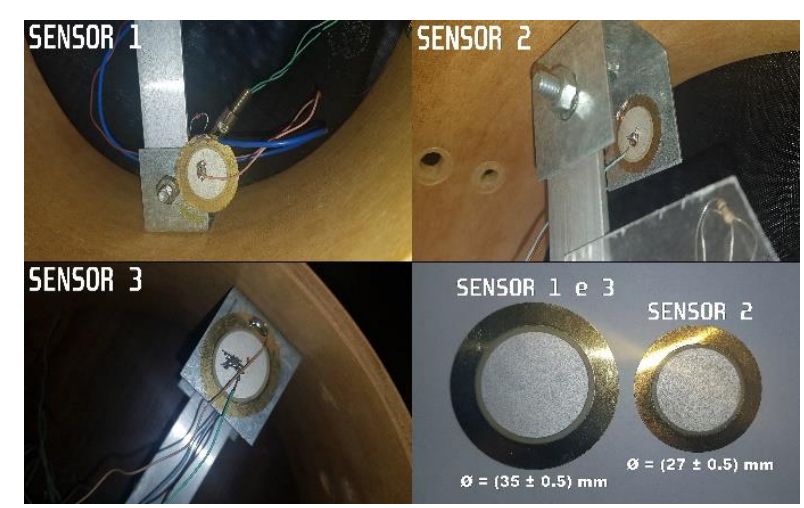

Figura 2: Foram testadas duas posições diferentes (sensores 1 e 2) e dois tamanhos diferentes (sensores $1 / 3$ e 2 ).

\subsection{Otimização do sinal}

Para melhor desempenho, fizemos testes com o intuito de otimizar o sinal recebido pelos sensores. O critério foi baseado na velocidade (kbit/s) do sinal elétrico, fornecido pelo software Hairless. Para o sensor do aro, variamos duas posições diferentes e dois tamanhos variados (Figura 2). Registramos os dados obtidos em 
cada combinação e analisamos qual demonstrava melhor sinal.

A membrana, como parâmetro, o parafuso ajustável (Figura 3, à direita), permitindo regular a altura da esponja (constituído de um tecido de nome Tatame, responsável por transmitir toda a pressão exercida na membrana sobre o sensor). $O$ objetivo era verificar qual a altura ideal para que o todos os pontos de pressão da membrana sejam captados pelo sensor. Para ambas as zonas, (Figura 3, à esquerda), o sinal que apresentasse maior intensidade nos diria qual caminho seguir.

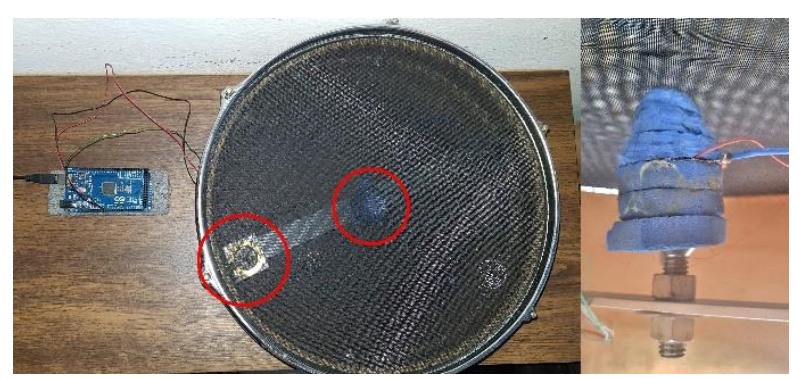

Figura 3: Esquerda - Tambor montado. Em destaque estão presentes o sensor do aro e em sequência o responsável pela membrana. Direita - Espuma responsável por transmitir a pressão exercida sobre a membrana ao sensor piezoelétrico.

\section{Resultados e Discussão}

Os sensores do aro foram submetidos a testes de intensidade. Foram desferidos vários impactos em pontos aleatórios da armação, anotamos os valores de velocidade (kbit/s) e realizamos a média dos dados obtidos. As batidas foram agrupadas em forças classificadas como Fraca, Média e Forte e a partir disso confeccionamos um gráfico (Figura 4). O resultado revelou que o sensor 2 apresentou melhor sinal.

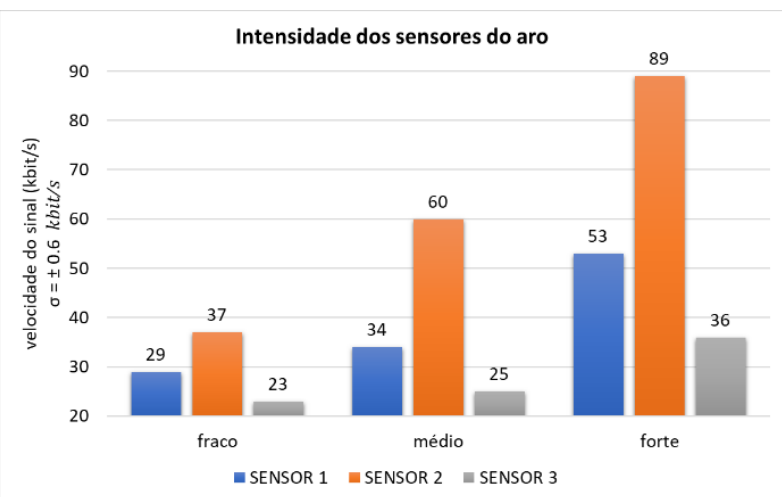

Figura 4: A combinação de posição e tamanho que apresentou melhor resultado para a batida no aro foi o sensor 2 .

Sobre a espuma que envolve o sensor da membrana (Figura 3, à esquerda), para a sua confecção experimentamos vários materiais de densidades variáveis, porém 0 tecido denominado Tatame demonstrou ser o mais eficaz. Os outros utilizados não demonstravam eficácia, pois parte da pressão exercida sobre eles era absorvida, em outras palavras, somente uma ínfima parte era transmitia para o sensor, não sendo suficiente para fazer o efeito de torção no elemento transdutor.

Para confecção, envolvemos o sensor com três camadas do tecido, formando um corpo oval, e abaixo mais três camadas. Utilizamos o formato que se assemelha a de um paraboloide circular pois sua geometria favorece que todos os pontos de pressão da membrana circular, sejam recebidos pelo material esponjoso, evitando a existência de regiões da pele do tambor ao qual o sensor não pudesse captar. As camadas abaixo foram colocas com o intuito de absorver as vibrações exercidas pelo aro, evitando que interfira no sinal da membrana.

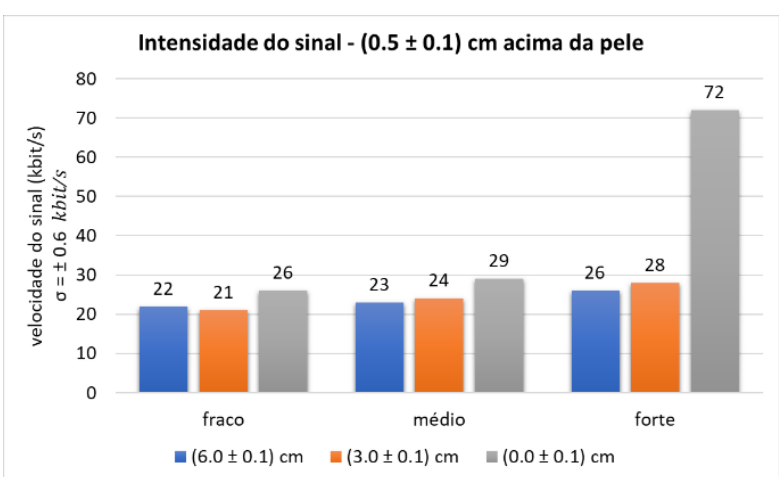

Figura 5: A distância ideal entre a ponta da espuma e a pele foi de 0.5 $\pm 0.1 \mathrm{~cm}$, uma vez que o sinal pode ser detectado para todos os níveis de pressão aplicada

Para garantir que todos os pontos de pressão da membrana pudessem ser transmitidos ao sensor, experimentamos várias alturas, reguladas pelo parafuso ajustável (figura 3, à direita). Isso nos garantiu um sinal otimizado. Os testes consistiam em realizar impactos sobre a membrana, utilizando os mesmos parâmetros de força, variando a distância da força aplicada de $(0.0 \pm 0.1) \mathrm{cm}$ a $(6.0 \pm 0.1) \mathrm{cm}$, tendo como origem o centro do tambor, sendo aro o limite. Essa sequência foi repetida para alturas variando de $(0.0 \pm$ $0.1) \mathrm{cm}$ até $(0.7 \pm 0.1) \mathrm{cm}$ acima da pele. Terminado os testes, calculamos a média das velocidades e comparamos cada uma. Nossa intenção era averiguar qual apresentava maior grau de intensidade.

A altura era medida a partir da ponta da espuma e a membrana, sendo a origem quando a membrana está totalmente esticada, e aumentando à medida que perfura a pele. Observamos que a distância adequada é $0.5 \pm 0.05 \mathrm{~cm}$, dado que foi a única que apresentava sinal em todos os pontos de pressões aplicados. O perfil de intensidade de velocidade é representado pela figura 5 .

\section{Agradecimentos}

Os autores agradecem à Universidade Federal do Espírito Santo pelo apoio por meio do Programa Institucional de Bolsas de Iniciação Científica (PIBIC) no período de 2019 a 2020. 


\section{Encontro Científico de Física Aplicada}

\section{Conclusão}

O tambor elétrico vai além de um instrumento de estudo para prática musical. Ele nos permite explorar um aprendizado voltado tanto para o estudo do hardware, quanto para software, proporcionando o estudo da informática envolvida, tal como a programação do microcontrolador e processamento de dados. Ademais, pelo efeito piezoelétrico presente no transdutor, conseguimos estudar conceitos pertinentes de Eletromagnetismo, tal como a formação de campos elétricos.

\section{Referências}

[1] GAUTSCHI, Gustav. Piezoelectric sensors. In: Piezoelectric Sensorics. Springer, Berlin, Heidelberg, 2002.
[2] SOUSA JR, ITAMAR V.; et. al. Física experimental com Arduino: ondas em uma corda tensionada. Revista Brasileira de Ensino de Física, v. 42, 2020.

[3] VIEIRA, M.; MENDES Junior, J.; PIRES, M.; STEVA JR, S. Dispositivo para análise de pressão plantar em palmilhas utilizando piezoelétricos de baixo custo. In Anais do XXV Congresso Brasileiro de Engenharia Biomédica, p. 536-539, 2016.

[4] LANNES, D. Transdutor Para Medição de deformações dinâmicas em dutos. PhD thesis, Dissertação (Mestrado em Engenharia Mecânica), Universidade Federal Fluminense - UFF, Niterói/RJ, 2009.

[5] REZENDE, Sergio Machado. Materiais e dispositivos eletrônicos. Editora Livraria da Física, 2004. 